\title{
Editorial
}

\section{Healthy Eating and Active Living in a Post-Pandemic World}

\author{
Jay E. Maddock ${ }^{1}$ and Rebecca Seguin-Fowler ${ }^{2}$ \\ ${ }^{1}$ Texas A\&M University, College Station, Texas \\ ${ }^{2}$ AgriLife Research, College Station, Texas
}

Just over a year ago, in February of 2020 at the Active Living Conference in Orlando, Florida, we made the first public announcement of the launch of a new journal entitled Journal of Healthy Eating and Active Living (JHEAL). At the time, none of us could have known what the next year (and beyond) would hold. The COVID-19 pandemic has had direct effects on active living through closures and limitations on access to gyms, parks, and greenspaces; social distancing regulations; and mask mandates. Overnight, something that millions of us took for granted, the ability to walk out the front door of our house or apartment and take a walk, carefree, now felt very different. In some places, it wasn't even allowed, and the intensity was exacerbated in dense urban areas like New York City, where shared public spaces like playgrounds for kids are the only option for families to get out and playand yet they were closed. Our food environments were dramatically altered as well, and not for the best. Our food supply chain, from production to distribution to retail, had been upended. Restaurants were closed, some permanently. In the early days, weeks, and even months, grocery store shelves were bare, lines were long, and the stress and anxiety were palpable.

The pandemic also starkly illustrated the inequities in access and outcomes. Those who could afford to hoard resources did so (when they could be found); those who had the space and money acquired their own exercise equipment while other people with personal yards and decks were able to access fresh air and greenspace in a way that was inaccessible to others. Older adults, who were among the very highest at risk, were shuttered in - away from the outdoors, socializing, and family. Concurrently, we rapidly understood the differential health effects of COVID-19 among Blacks, Hispanics, and Native Americans: These Americans were twice as likely to die from the disease as White Americans (CDC, 2021). While cases by race and ethnicity were only somewhat elevated (1.1-1.7 times), the number of hospitalizations and severe and fatal cases were much higher (1.9-3.7 times; CDC, 2021). Many of the preexisting conditions that are related to severe cases of COVID-19, including obesity, heart disease, type 2 diabetes, and hypertension (CDC, 2020), are impacted by healthy eating and active living - things that the pandemic was wreaking havoc on. Within this context, food deserts continued to persist, affecting more than $5 \%$ of U.S. Census tracts and occurring more frequently in lowincome communities of color (Karpyn et al., 2019). Similarly, access to parks and greenspaces is also lower in these communities (Wen et al., 2013), exacerbating the effects of the pandemic on health and well-being.

As vaccinations are starting to become more widespread, there appears to be a light at the end of the tunnel, although "end" is a relative term in this case. Pandemics are far from over; we are in an interpandemic, or between pandemic, phase. With the increase in human populations and development in previously wild areas, new infectious diseases will continue to appear. As the world opens back up, this is a unique time to research and advocate for what we want the new normal to be. Active Living Research, and now the Active Living Conference, have continually focused on bringing together researchers with elected officials, public works, city planners, and other practitioners to focus on creating active living communities. The pandemic has only increased the need for these groups to work together to create a more equitable world. And yet several of our prior understandings about active living and healthy eating environments have also been challenged. In a pre-COVID-19 world, dense mixeduse developments, public transportation, bike shares, and reducing urban sprawl appeared to be positive, overall, for promoting active living and improving access to healthy eating and related resources. For some, that reality had different impacts during an infectious disease outbreakparticularly one lasting such a long time. How will features and assets be viewed in the proximal years following COVID-19? How will the food system adapt? How will people consider density, greenspace, and shared usage of parks, transportation, and other resources even when "normal" is getting closer in reach.

Importantly, those considerations assume, to some degree, that people have choices in the location, housing, and communities they live in, which we know isn't always true. This pandemic has starkly illustrated disparities present in our communities, across the social determinants of health. What lessons in health and beyond can we use to challenge previous assumptions and create meaningful and sustainable change? How can we open up to a more just and equitable world?

Over the next year, as the world approaches herd immunity and COVID-19 finally starts to wane, life for everyone won't (and optimally shouldn't) return to exactly as it was pre-COVID-19. This presents a challenge for us to address in research and policy efforts to create a more resilient, equitable world in which basic human rights include stable access to nutritious and affordable foods; diverse greenspaces with clean air and access for various 
abilities; and access to quality health care services, including prevention. We look forward to reading about these in JHEAL.

Corresponding Author:

Jay E. Maddock, Ph.D.

School of Public Health

Texas A\&M University

College Station, TX 77843

maddock@tamu.edu

1-979-436-9346

Jay E. Maddock 0000-0002-1119-0300

iD $5115-2341$

\section{Author Contributions}

Conceptualization, J.E.M. and R.S.F.; Writing-Original Draft, J.E.M.; Writing - Reviewing \& Editing, J.E.M. and R.S.F.

\section{Conflicts of Interest}

The authors report no conflicts of interest.

\section{Creative Commons License:}

This work is licensed under a Creative Commons Attribution-Noncommercial 4.0 International License (CC BY-NC 4.0).

Suggested citation (APA 7th edition): Maddock, J.E. \& Seguin-Fowler, R. (2021). Healthy eating and active living in a post-pandemic world. Journal of Healthy Eating and Active Living, 1(2), 39-40.

\section{References}

Centers for Disease Control and Prevention. (2020). Evidence used to update the list of underlying medical conditions that increase a person's risk of severe illness from COVID-19. Accessed on-line at https://www.cdc.gov/coronavirus/2019ncov/need-extra-precautions/evidence-table.html on March 16, 2021.

Centers for Disease Control and Prevention. (2021). Risk for COVID-19 infection, hospitalizations and death by race/ethnicity. Accessed March 16, 2021 at https://www.cdc.gov/coronavirus/2019-ncov/covid-data/investigationsdiscovery/hospitalization-death-by-race-ethnicity.html.

Karpyn, A. E., Riser, D., Tracy, T., Wang, R., \& Shen, Y. (2019). The changing landscape of food deserts. UNSCN Nutrition, $44: 46-53$.

Wen, M., Zhang, X., Harris, C. D., Holt, J. B., \& Croft, J. B. (2013). Spatial disparities in the distribution of parks and green spaces in the U.S. Annals of Behavioral Medicine, 45:18-27. 